\title{
Crossed Cerebellar Diaschisis in Status Epilepticus
}

\author{
Daigo Miyazaki ${ }^{1}$, Kazuhiro Fukushima ${ }^{2}$, Asa Nakahara ${ }^{1,3}$, Minori Kodaira ${ }^{1}$, \\ Katsunori Mochizuki ${ }^{4}$, Kikuko Kaneko ${ }^{5}$, Tomoki Kaneko ${ }^{5}$, \\ Yoshiki Sekijima $^{1}$ and Shu-ichi Ikeda ${ }^{1}$
}

\begin{abstract}
Crossed cerebellar diaschisis (CCD) is an interesting phenomenon which classically refers to the depressed blood flow and metabolism affecting one cerebellar hemisphere after a contralateral hemispheric infarction. However, CCD can also be caused by a prolonged seizure. We herein report a case of CCD due to status epilepticus in a patient who showed unique magnetic resonance imaging findings.
\end{abstract}

Key words: crossed cerebellar diaschisis, status epilepticus

(Intern Med 55: 1649-1651, 2016)

(DOI: 10.2169/internalmedicine.55.6689)

\section{Case Report}

A 67-year-old man with a history of alcoholism was transferred to our hospital with convulsive status epilepticus (SE). Three months earlier, he had caused a traffic accident while driving to the neighboring prefecture and was admitted to the nearest general hospital. He had no apparent traumatic injuries but experienced a single convulsive seizure in the emergency room. He was discharged with a recommendation to consult a physician at his local hospital, which he did not follow.

On admission, he presented with repetitive generalized tonic-clonic seizures that first affected the right side of his face and upper right arm. His Glasgow coma scale (GCS) score was E1V2M4. A physical examination revealed that his blood pressure was $112 / 88 \mathrm{~mm} \mathrm{Hg}$, his heart rate was $153 \mathrm{bpm}$, and his body temperature was $39.4^{\circ} \mathrm{C}$. Brain computed tomography only revealed mild cerebral atrophy. His blood tests results were as follows: hemoglobin, $17.0 \mathrm{~g} / \mathrm{dL}$; white blood cell count, $12,550 / \mathrm{mm}^{3}$; platelets, $132,000 / \mathrm{mm}^{3}$; serum total bilirubin, $2.60 \mathrm{mg} / \mathrm{dL}$; aspartate aminotransferase, 259 IU/L; alanine aminotransferase, $88 \mathrm{IU} / \mathrm{L} ; \gamma$ glutamyl transpeptidase, 1,376 IU/L; creatinine phosphok- inase, $976 \mathrm{IU} / \mathrm{L}$; albumin, $3.5 \mathrm{~g} / \mathrm{L}$; creatinine, $1.52 \mathrm{mg} / \mathrm{dL}$; and blood urea nitrogen, $17.8 \mathrm{mg} / \mathrm{dL}$. His blood levels of vitamin B1, vitamin B12, and folic acid were normal $(4.1 \mu \mathrm{g} /$ $\mathrm{dL}, 670 \mathrm{pg} / \mathrm{mL}$, and $5.1 \mathrm{ng} / \mathrm{mL}$, respectively). A cerebrospinal fluid (CSF) analysis showed a normal cell count and glucose level and a moderately elevated total protein concentration $(75 \mathrm{mg} / \mathrm{dL}$, normal range: $10-40 \mathrm{mg} / \mathrm{dL})$. A polymerase chain reaction test for herpes simplex virus in CSF was negative. Artificial ventilation and the intravenous administration of midazolam and fosphenytoin sodium hydrate were initiated, but his convulsive seizure was intractable. Electroencephalography revealed left periodic lateralized epileptiform discharges characterized by rhythmic delta waves over the left frontal, central, and mid-temporal regions (Fig. 1). On brain magnetic resonance imaging (MRI), fluid-attenuated inversion recovery (FLAIR) and diffusionweighted images (DWI) revealed marked hyperintensity and gyral swelling in the cortex of the left hemisphere and the contralateral cerebellum with a corresponding low apparent diffusion coefficient (ADC) (Fig. 2A). Increased flow in the branches of the left middle cerebral artery (MCA) was evident on magnetic resonance angiography (Fig. 3). These findings were consistent with left hemispheric status epilepticus with crossed cerebellar diaschisis (CCD). The DWI

\footnotetext{
${ }^{1}$ Department of Medicine (Neurology and Rheumatology), Shinshu University School of Medicine, Japan, ${ }^{2}$ Department of Home Care Promotion, Shinshu University School of Medicine, Japan, ${ }^{3}$ Clinical Training Center, Shinshu University Hospital, Shinshu University School of Medicine, Japan, ${ }^{4}$ Department of Emergency and Critical Care Medicine, Shinshu University School of Medicine, Japan and ${ }^{5}$ Department of Radiology, Shinshu University School of Medicine, Japan

Received for publication October 27, 2015; Accepted for publication December 23, 2015
}

Correspondence to Dr. Kazuhiro Fukushima, fukushima-k@umin.org 


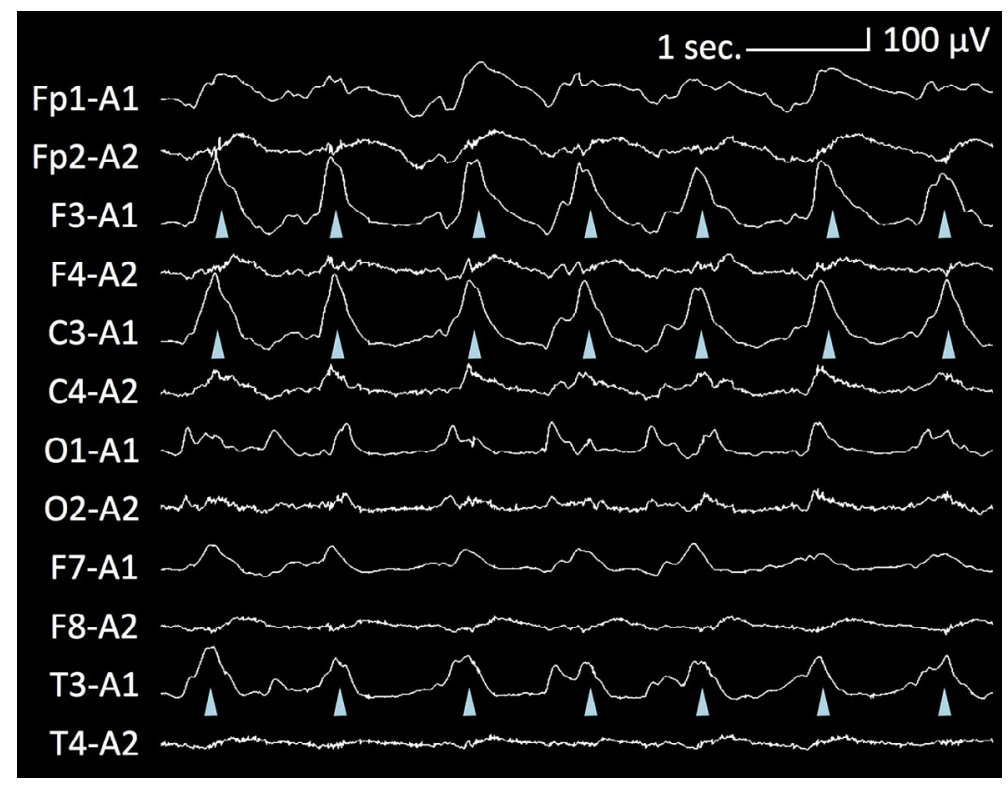

Figure 1. Electroencephalography performed on admission showed left periodic lateralized epileptiform discharge predominantly in the left frontal, central, and mid-temporal regions (arrowheads).

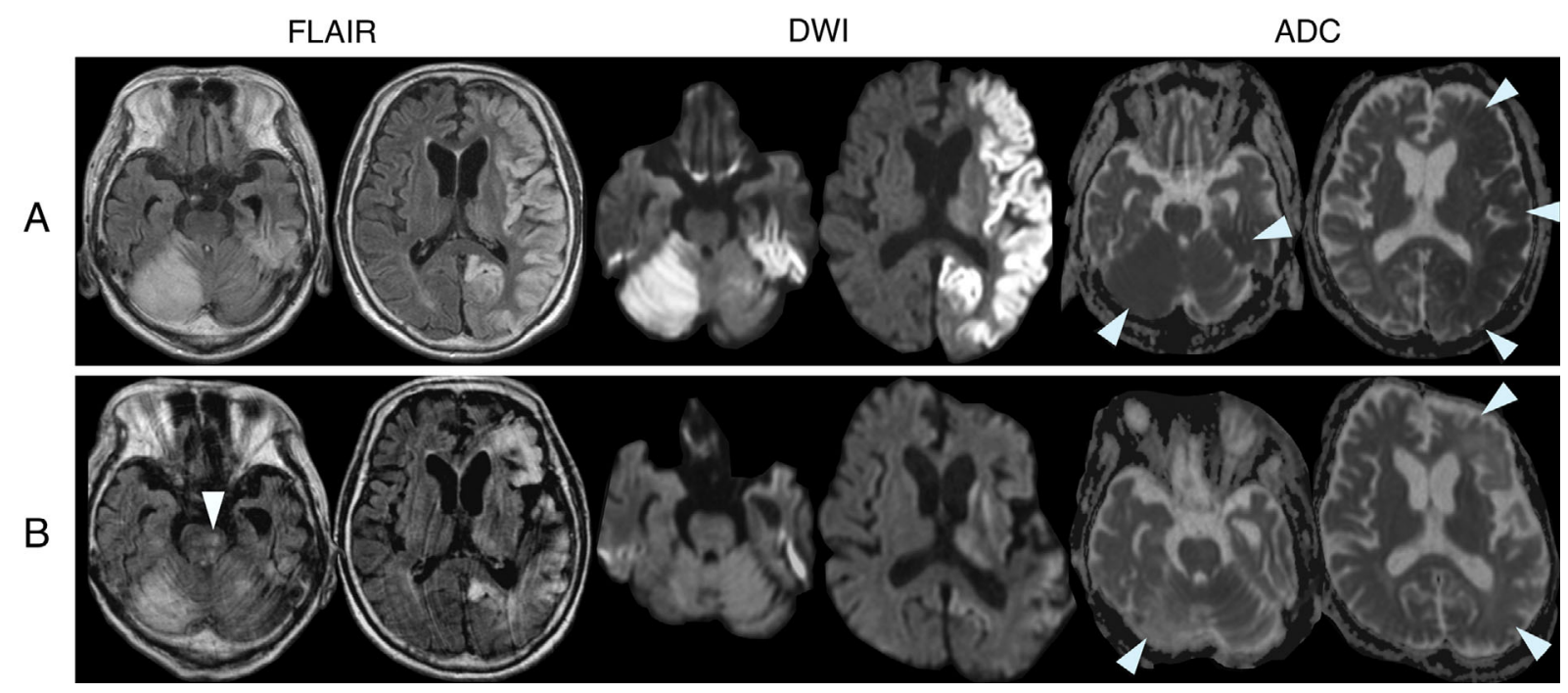

Figure 2. Brain magnetic resonance imaging (MRI) on days 5 (A) and 46 (B) after admission. A: Fluid-attenuated inversion recovery (FLAIR) and diffusion-weighted images (DWI) showed cortical hyperintensity with gyral swelling in the left hemisphere and contralateral cerebellum, corresponding to areas with a low apparent diffusion coefficient (ADC) (arrowheads). B: Follow-up DWI revealed reduced cortical hyperintensity and gyral swelling in the left and contralateral cerebellum, as well as brain atrophy with high ADC (arrowheads) and sustained hyperintensity on FLAIR MRI. FLAIR MRI revealed a new area of high intensity in the left basilar part of the pons (arrowhead), corresponding to the pontine loci of cortico-ponto-cerebellar (CPC) pathway. The movement of the patient generated motion artefacts, especially in the follow-up scans.

findings showed the restricted diffusion of water molecules, and indicated cytotoxic damage. The patient's SE ceased on the fifth day of admission, but he had experienced severe brain damage.

Follow-up MRI was performed 41 days after the initial imaging session. The gyral swelling was observed to have decreased in both the left hemisphere and the contralateral cerebellum, but distinct brain atrophy was noted. ADC hyperintensity with sustained short tau inversion recovery (STIR) hyperintensity was observed, but the DWI hyperintensity had disappeared (Fig. 2B). These findings were compatible with postictal brain atrophy followed by crossed cerebellar atrophy (CCA). The DWI and ADC signal alterations indicated a change from cytotoxic to vasogenic edema. 

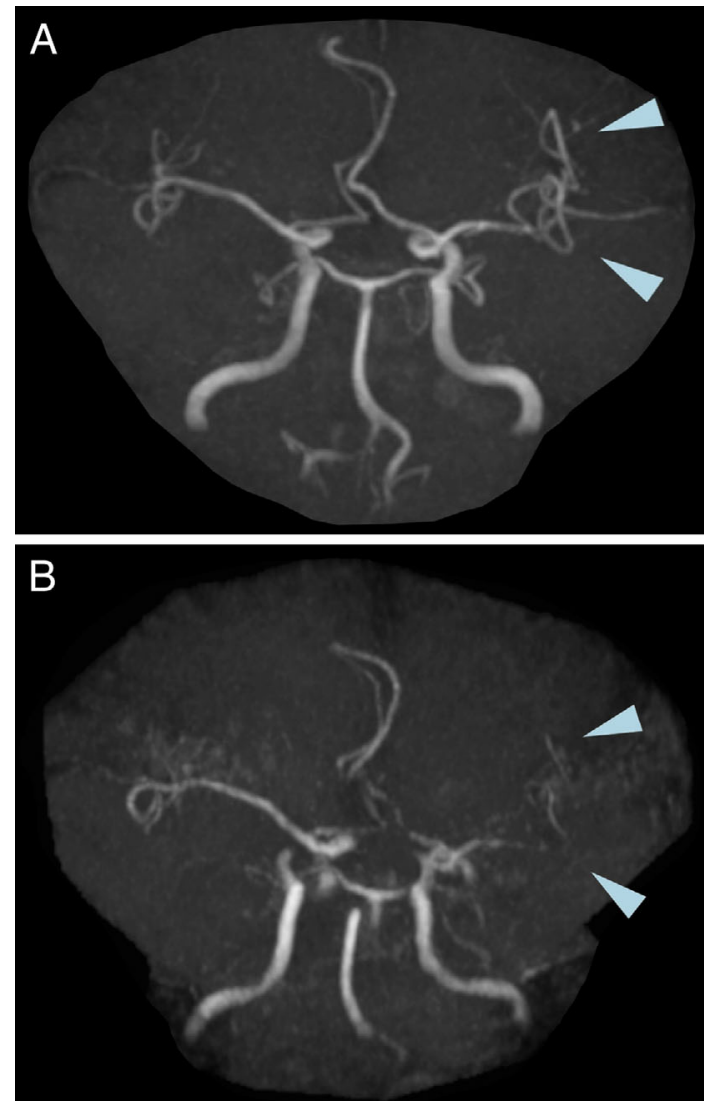

Figure 3. Magnetic resonance angiography (MRA) on days 5 (A) and 46 (B) after admission. The initial MRA showed the vasodilation of the left middle cerebral artery (MCA) branches (A, arrowheads). Decreased flow in the branches of the left MCA was also observed on follow-up MRA (B, arrowheads). The movement of the patient generated motion artefacts in the follow-up scans.

On FLAIR MRI, a new area of high intensity was observed in the left basilar part of the pons, corresponding to the pontine loci of the cortico-ponto-cerebellar (CPC) pathway (Fig. 2B). On MRA, decreased flow was also noted in the branches of the left MCA, which might have reflected the resolution of ictal hyperperfusion and the atrophy of the left cerebral hemisphere (Fig. 3).

\begin{tabular}{l} 
Discussion \\
\hline
\end{tabular}

The cerebellum receives significant input from the cerebral hemispheres via the CPC pathway. Conventionally, CCD refers to the depression of blood flow and metabolism affecting the cerebellar hemisphere due to a contralateral focal, supratentorial lesion, such as a cerebral infarction or tumor $(1,2)$. Prolonged seizure activity in SE can uncouple the metabolism and the cerebral blood flow, resulting in tissue hypoxia, anaerobic glycolysis, sodium/potassium pump failure, and cytotoxic edema (3). CCD following SE is caused by excessive neuronal transmission from the prolongation of excitatory synaptic activity via the CPC pathway (3-5). A few cases of CCA after CCD have been reported. Diffuse neuronal loss in the affected and contralateral cerebral hemispheres and the degeneration of the pontine nuclei and CPC fibers have been reported as pathological findings in patients with CCA (5). Repetitive seizures and SE could be a main pathogenic contributor in CCA. Seizure-related trans-synaptic excitotoxic cell damage causes irreversible structural changes (1).

The authors state that they have no Conflict of Interest (COI).

\section{References}

1. Tien RD, Ashdown BC. Crossed cerebellar diaschisis and crossed cerebellar atrophy: correlation of MR findings, clinical symptoms, and supratentorial diseases in 26 patients. AJR Am J Roentgenol 158: 1155-1159, 1992.

2. Huang YC, Weng HH, Tsai YT, et al. Periictal magnetic resonance imaging in status epilepticus. Epilepsy Res 86: 72-81, 2009.

3. Samaniego EA, Stuckert E, Fischbein N, et al. Crossed cerebellar diaschisis in status epilepticus. Neurocrit Care 12: 88-90, 2010.

4. Koy A, Klee D, Weber AA, et al. Crossed cerebellar diaschisis after status epilepticus in a young child. Neuropediatrics 43: 55-58, 2012.

5. Tan N, Urich H. Postictal cerebral hemiatrophy: with a contribution to the problem of crossed cerebellar atrophy. Acta Neuropathol (Berl) 62: 332-339, 1984.

\footnotetext{
(C) 2016 The Japanese Society of Internal Medicine http://www.naika.or.jp/imonline/index.html
} 